\title{
EFFECTS OF RESVERATROL AND MORIN ON INSOLUBLE TAU IN TAU TRANSGENIC MICE
} are neurodegenerative diseases, including Alzheimer's disease (AD) and frontotemporal dementia (FTD), in which tau protein aggregates within neurons. An effective treatment is lacking and is urgently needed. We evaluated two structurally similar natural compounds, morin and resveratrol, for treating tauopathy in JNPL3 P301L mutant human tau overexpressing mice. Rotarod tests were performed to determine effects on motor function. After treatment from age 11 to 14 months, brains of 26 mice were collected to quantify aggregated hyperphosphorylated tau by Thioflavin $\mathrm{T}$ and immunohistochemistry (IHC) and to quantify total tau (HT7 antibody) and hyperphosphorylated tau (AT8 antibody) in homogenates and a fraction enriched for paired helical filaments. Resveratrol reduced the level of total hyperphosphorylated tau in IHC sections $(p=0.036)$, and morin exhibited a tendency to do so $(p=0.29)$, while the two drugs tended to increase the proportion of solubilizable tau that was hyperphosphorylated, as detected in blots. Neither resveratrol nor morin affected motor function. One explanation of these results is that the drugs might interrupt a late stage in tau aggregation, after small aggregates have formed but before further aggregation has occurred. Further animal studies would be informative to explore the possible efficacy of morin or resveratrol for treating tauopathies.

Keywords - tauopathy • Alzheimer's disease • frontotemporal dementia • neurofibrillary tangle - neurodegeneration • paired helical filament • flavonoid

\section{Introduction}

The microtubule associated protein tau promotes assembly and stability of microtubules [1]. Hyperphosphorylation of tau reduces its ability to fulfill this function, leading to microtubule disruption and to sequestration of tau in small aggregates called oligomers or larger aggregates called paired helical filaments (PHF), which in turn clump together to form neurofibrillary tangles (NFT) [1]. Oligomers of $A \beta$ peptide or tau may be more toxic to neurons than are plaques or tangles [2]. Tangles occur in a variety of neurodegenerative diseases, including Alzheimer's disease (AD), frontotemporal dementia, and progressive supranuclear palsy. In $A D$, the commonest tauopathy, the appearance of aggregated tau likely comes after $A \beta$ aggregation and toxicity, which can lead to hyperphosphorylation of tau via activation of particular kinases [3].

Recent clinical trials of potential $A D$ drugs that aim to decrease $A \beta$ levels have failed to slow progression in $A D$ patients, raising suggestions that, to prevent neurodegeneration, treatment that targets $A \beta$ may need to begin at an earlier stage in the course of the disease [4]. However, it is difficult to identify patients before they display memory deficits. An alternative strategy would target $A D$ pathways downstream of $A \beta$, such as tau aggregation perhaps. Increasing evidence suggests that abnormal aggregates of tau travel between cells and initiate further aggregation of tau in a spreading network of neurons [5], thus another advantage of targeting tau aggregation would be the opportunity to interrupt this trans-neuronal process.

Many compounds, both natural and synthetic, have been found to inhibit tau aggregation $[6,7]$. One such molecule is morin, a naturally occurring flavonoid which can be found in some plants, including old fustic, osage orange, guava, onion, and apple [8]. The chemical structure of morin (Figure 1) is similar to that of other flavonoids such as myricetin and
Kwun Chung Yu',

Ping Kwan ${ }^{1,2}$,

Stanley K.K. Cheung ${ }^{3}$,

Amy $\mathrm{Ho}^{4}$,

Larry Baum ${ }^{5 *}$

'School of Pharmacy, The Chinese University of Hong Kong, Shatin, Hong Kong

2Level 5, R.M.C. Gunn Building, The University of Sydney, Sydney, NSW 2006, Australia

${ }^{3}$ School of Biomedical Sciences, The Chinese University of Hong Kong, Shatin, Hong Kong

${ }^{4}$ Department of Anatomy, The Chinese University of Hong Kong, Shatin, Hong Kong

The State Key Laboratory of Brain and Cognitive Sciences, University of Hong Kong, Pokfulam, Hong Kong; Centre for Genomic Sciences, University of Hong Kong, Pokfulam, Hong Kong

Received 25 February 2018 accepted 10 May 2018 gossypetin, which inhibited tau aggregation in vitro with half maximal inhibitory concentrations (IC50s) of approximately 1-2 $\mu \mathrm{M}[9,10]$. Morin inhibited tau aggregation in an in vitro assay with an IC50 of approximately $13 \mu \mathrm{M}$ (PubChem BioAssay AID 1460, CID 5281670). In vivo, morin can inhibit an enzyme that phosphorylates tau, glycogen synthase kinase 3 beta (GSK3 $\beta$ ), and thus decrease tau hyperphosphorylation [8].

Resveratrol, an antioxidant polyphenol, has a structure (Figure 1) related to that of the flavonoids mentioned above. Resveratrol promoted clearance of $A \beta$ peptide [11-14] and inhibited tau aggregation in an in vitro assay with an IC50 of approximately $10 \mu \mathrm{M}$ (PubChem BioAssay AID 1460, CID 445154). Like morin, resveratrol inhibited GSK3 $\beta$ in vivo and decreased brain levels of phosphorylated tau (ptau) in one study, though not in another study $[15,16]$. In transgenic mice overexpressing P301 L mutant tau as a model of frontotemporal dementia, we examined the potential for morin 
and resveratrol to prevent tau aggregation and deterioration in motor function.

\section{Methods}

\subsection{Transgenic mice}

JNPL3 tau transgenic mice were purchased from Taconic Farms, Inc. and were bred at the Chinese University of Hong Kong for experiments [17]. The mice overexpress two copies of the $4 R 0 N$ isoform of the P301L mutant human microtubule-associated protein tau to serve as a model of tauopathies, including AD. At age 11 months, 27 mice were randomly assigned to three groups of equal size for treatment with morin, resveratrol, or placebo. Per gram of body weight, $0.1 \mathrm{mg}$ of drug (or nothing for the placebo group) was mixed with $1 \mu \mathrm{L}$ peanut butter and injected by syringe without a needle onto the mouth once daily from Monday to Friday. Mice readily ate all the peanut butter, thus ensuring full compliance in consuming the entire dose of drug. This non-invasive delivery method prevented injury to mice and modeled an oral mode of administration that may be used in human treatment.

To minimize bias, all the experiments involved in this study were performed in a blind fashion, with identification of the treatment of individual mice unknown by the person performing laboratory analysis until quantification of all mice had been completed.

\subsection{Ethics Statement}

All experimental protocols were approved by the Chinese University of Hong Kong Animal Experimentation Ethics Committee (Ref No. 07/043/ERG and 460207). Several mice were<smiles>O=c1c(O)c(-c2ccc(O)cc2O)oc2cc(O)cc(O)c12</smiles>

Morin housed in each cage and had free access to food and water.

\subsection{Sample Preparation}

After treatment for three months, mice were euthanized by isoflurane, cardiac perfused with ice-cold PBS, and brains were removed. One mouse died before the end of the treatment period, thus only 26 brains were collected. One hemisphere from each mouse was frozen at $-80^{\circ} \mathrm{C}$ for immunoblotting, and the other hemisphere was fixed in $10 \%$ formalin in PBS overnight, embedded in paraffin and cut into 5 micrometer sagittal sections using a microtome.

\subsection{Immunoblotting}

Homogenization buffer contained $25 \mathrm{mM}$ Tris pH 7.4, 1 mM EDTA, 1 mM EGTA, 150 mM $\mathrm{NaCl}, 1 \%$ phosphatase inhibitor cocktail (Sigma P0044 and Sigma P5726), and 1X Complete, EDTA-free Protease Inhibitor Cocktail (Roche 11873580 001). Each brain hemisphere was weighed and Dounce homogenized with $3 x$ (volume/weight) ice cold homogenization buffer. Protein content of the homogenate was measured by bicinchoninic acid assay, and paired helical filaments (PHF) were enriched in half of the homogenate by the following procedure. Homogenates (volumes were measured: $\mathrm{V} \mathrm{ml}$ ) were incubated on ice for 20 minutes to depolymerize microtubules and were then centrifuged at $80,000 \mathrm{~g}$ for 15 minutes at $4^{\circ} \mathrm{C}$. The pellet was homogenized using a Dounce homogenizer in $\mathrm{V} \mathrm{ml}$ of A68 extraction buffer $(10 \mathrm{mM}$ Tris $\mathrm{pH} 7.4$, $1 \mathrm{mM}$ EGTA, $0.8 \mathrm{M} \mathrm{NaCl}, 10 \%$ sucrose, $1 \%$ phosphatase inhibitor cocktail [Sigma P0044<smiles>Oc1ccc(/C=C/c2cc(O)cc(O)c2)cc1</smiles>

Resveratrol and Sigma P5726], and 1X Complete, EDTAfree Protease Inhibitor Cocktail [Roche 11873 580 001]) and centrifuged at $1000 \mathrm{~g}$ for 10 minutes at $4^{\circ} \mathrm{C}$. The resulting supernatant was transferred to a clean tube, and sarkosyl was added to $1 \%$ for incubation on a rotating wheel for 1.5 hours at room temperature, followed by centrifugation at $80,000 \mathrm{~g}$ for 30 minutes at $4^{\circ} \mathrm{C}$ and concluding with resuspension of the pellet in $0.2 \mathrm{~V}$ homogenization buffer.

Homogenates containing equal volumes of protein, or PHF fractions extracted from a fixed multiple of those volumes of homogenates, were immunoblotted to measure total tau (Thermo Scientific, Cat.\#MN1000, 1:5000 HT7 anti-human tau monoclonal antibody) [20], hyperphosphorylated tau (Thermo Scientific, Cat.\# MN1080, 1:1000 AT8 anti-human PHF tau monoclonal antibody, which recognizes tau phosphorylated at Ser199 and Ser202), and GAPDH (Sigma G8795, 1:10000 antiGAPDH monoclonal antibody) [19]. Blots were incubated in antibodies diluted in Tris buffered saline Tween $+5 \%$ milk. Blots were photographed with an Alpha Innotech FluorChemQ Multilmage III, and protein bands were quantified using ImageJ software. Three gels were required to run all the samples, and one lane on each gel was reserved to run a sample duplicated from another gel for normalization of protein bands. Quantitation of total tau and hyperphosphorylated tau were measured and normalized to GAPDH.

\section{5.. Thioflavin T Staining}

Paraffin sections were stained using Thioflavin $T$ to detect tangles [18]. First, sections were deparaffinized for one hour at $60^{\circ} \mathrm{C}$ and immersed in xylene thrice for 5 minutes to remove paraffin. They were rehydrated in descending concentrations of ethanol in water, then immersed for 5 minutes in Mayer's hematoxylin. Sections were then washed in running tap water for 5 minutes and then rinsed in distilled water. Sections were immersed for 5 minutes in freshly prepared $1 \%$ Thioflavin T. Then the sections were differentiated in $70 \%$ ethanol for 5 minutes. Finally, they were rinsed in distilled water twice, mounted in glycerin jelly, and covered with cover slips.

Figure 1. Chemical structures of morin and resveratrol. 


\subsection{Immunohistochemistry}

Sections on slides were deparaffinized for one hour, placed in xylene and rehydrated in descending concentrations of ethanol in purified water. Antigen retrieval was then performed with boiling citrate buffer $(2.1 \mathrm{~g}$ citric acid monohydrate in $1 \mathrm{~L}$ water adjusted to $\mathrm{pH} 6.0$ with $\mathrm{NaOH}$ ) for half an hour.

Slides were rinsed with phosphate buffered saline (PBS) at $\mathrm{pH} 7.4$ (PBS) and were then immersed in 3\% hydrogen peroxide in water for 6 minutes to quench endogenous peroxidase activity.

Sections were blocked in 10\% normal goat serum in PBS, then incubated with AT8, a monoclonal antibody against a phosphorylated epitope of tau, (Thermo Scientific, Cat.\# MN1080, 1:100) overnight at room temperature [19]. Goat anti-mouse IgG antibody coupled to horseradish peroxidase polymer (DAKO Envision System, Cat.\#K4001) was added to the sections for 45 minutes. Sections were developed using 3,3'-diaminobenzidine (DAB) peroxidase solution and were counterstained by immersion in Harris Hematoxylin, dehydrated, mounted with Permount, and covered with cover slips.

\subsection{Imaging and Quantification}

"Q-capturing system" software was used for image processing. Randomly selected regions throughout the brain were photographed at 40x magnification, either by fluorescence microscopy for Thioflavin T staining or by bright field microscopy for AT8 immunostaining. Stained regions in the photos were recognized by setting a colour threshold and quantified by number of positively stained pixels. For each mouse, positive staining for all photos was averaged.

\subsection{Rotarod Test}

Motor function performance of mice was assessed by the rotarod test, using a protocol modified from EMPReSS (European Mouse Phenotyping Resource for Standardized Screens, at http://www.empress.har.mrc.ac.uk/ viewempress/?pipelineprocedure=EUMODIC +Pipeline+2 Rotarod). Both before and after treatment, one round of trial training and five rounds of testing were performed with a one hour interval in between. Mice were placed onto the rod of the apparatus, which was rotated at a constant rate of 4 revolutions per minute (rpm) and then accelerated at a rate of about $1 \mathrm{rpm} / 9$ seconds, to a maximum of 40 $\mathrm{rpm}$. The time between the beginning of the acceleration of the rod and the falling of each mouse was recorded. Mean times for each mouse at the end of treatment was calculated and compared among treatment groups.

\subsection{Statistics}

ANOVA tests were performed to compare all three treatment groups for rotarod testing immunohistochemistry, immunoblotting, and Thioflavin staining. Two-tailed t-tests were performed to compare control and treatment groups. Statistical analyses were conducted using Microsoft Office Excel 2007 or PSPP (gnu. org).

\section{Results}

We chose to test two drugs: morin and resveratrol. Morin was an attractive candidate because it is abundant in common foods and because it inhibits tau aggregation and GSK3 $\beta$, a tau kinase. Like morin, resveratrol inhibits tau aggregation and GSK3 $\beta$; it also induces removal of $A \beta$ peptide.

Nine mice completed treatment with morin eight with resveratrol (one mouse died before the end of the treatment period), and nine with no drug. The effects of treatment with morin or resveratrol on tau, hyperphosphorylated tau (ptau), and tangles are shown in Figures 2 and 3.

To measure the effect of the compounds on tau, ptau, and tangles, we used immunoblotting. Immunoblotting of total brain homogenate or a PHF-enriched brain fraction revealed no significant differences (by ANOVA) between treatments in either total tau (HT7 antibody), hyperphosphorylated tau (AT8 antibody), or the ratio of hyperphosphorylated tau to total tau (Figure 2). However, there appears to be a tendency toward increased hyperphosphorylated tau in the PHF fraction due to treatment with either drug (Figure $2 \mathrm{~g}$ ). To confirm this observation, a t-test (2-tailed, equal variances not assumed) was performed to compare control brains with drug-treated brains (pooling morin and resveratrol groups together), demonstrating a significant difference $(p=0.006)$. Similarly, the ratio of hyperphosphorylated tau to total tau (Figure 2i) was also increased in all drugtreated brains $(p=0.03)$. For total homogenate, the corresponding comparisons were $\mathrm{p}=0.035$ and $\mathrm{p}=0.84$ for hyperphosphorylated tau and the ratio of hyperphosphorylated to total tau, respectively. Therefore, the drugs increased the amount of extractable hyperphosphorylated tau.

To measure the effect of the compounds on tangles, we used immunohistochemistry. Immunohistochemistry suggested that morin and resveratrol exhibited a tendency to decrease tangle deposition (Figure 3 ). This tendency was stronger for resveratrol than for morin. The area of Thioflavin $T$ staining (not shown) did not significantly differ among the three treatment groups (Figure 3c, $p=0.42$ ), but the area of AT8 immunoreactivity (representative photos shown in Figure 3a) differed (Figure $3 b, p=0.045$ ). When comparing morin with control treatment, morin changed neither AT8 $(p=0.29)$ nor Thioflavin T $(p=0.57)$ staining. When comparing resveratrol with control treatment, resveratrol significantly altered AT8 $(p=0.036)$ but not Thioflavin T $(p=0.26)$ staining. Thus, resveratrol decreased tangles as measured by immunoreactivity.

Since tauopathies may affect motor function, we used the rotarod test. The effects of treatment with morin or resveratrol on motor coordination, as measured by the rotarod test, are shown in Figure 4. Rotarod performance did not significantly differ among the three treatment groups $(p=0.85)$. Therefore, the drugs did not affect motor function.

\section{Discussion}

Resveratrol and morin tended to increase hyperphosphorylated tau in the PHF fraction extracted from brain homogenates, while they tended to decrease hyperphosphorylated tau in tangles in brain sections. These apparently contradictory results are surprising. One possible explanation for the results is that these compounds might block or reverse late 
stages of aggregation of hyperphosphorylated tau, thus decreasing tangles but leading to accumulation (just before the point of blockage) of relatively more soluble forms of hyperphosphorylated tau. In other words, tau that has been hyperphosphorylated may bind resveratrol or morin and be stabilized in a relatively soluble form, preventing tau from aggregating into tangles. This would also have the effect of increasing the amount of extractable hyperphosphorylated tau. Conversely, the absence of resveratrol or morin might allow hyperphosphorylated tau to aggregate into insoluble tangles, thus increasing tangles visible in brain sections and

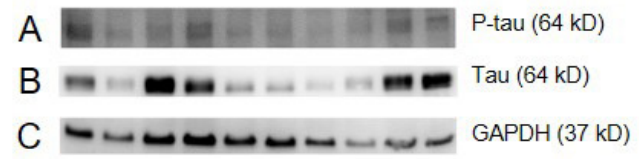

D Homogenate AT8 immunoreactivity

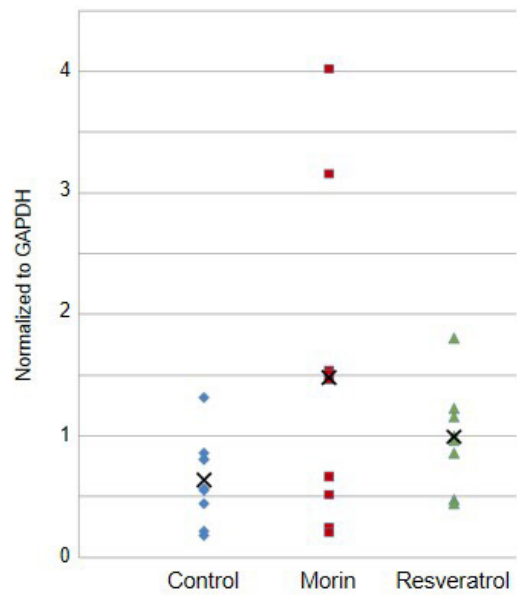

G

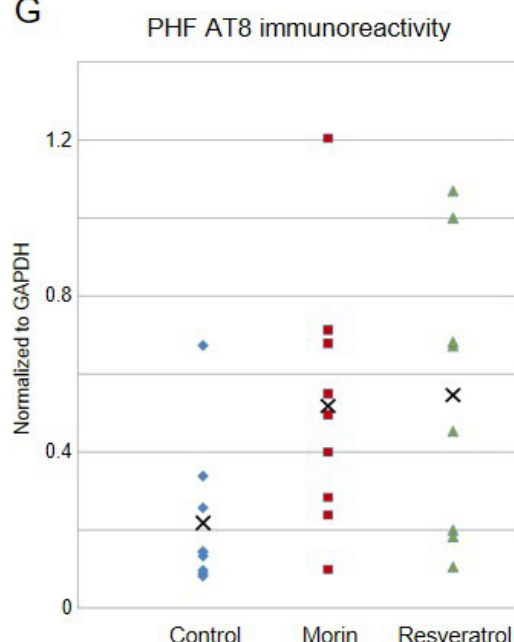

$E$

Homogenate HT7 immunoreactivity

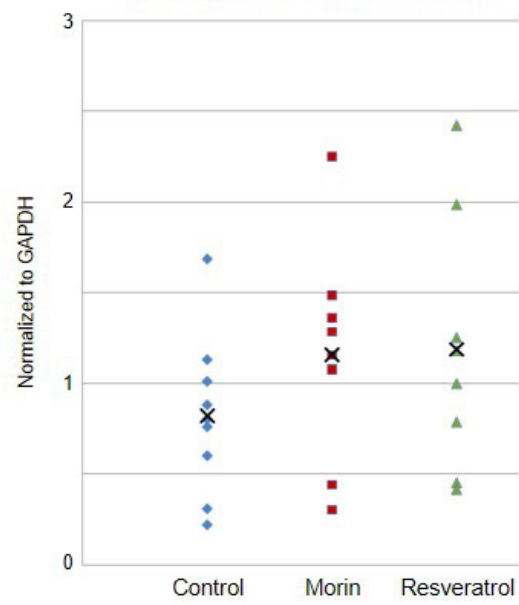

$\mathrm{H}$

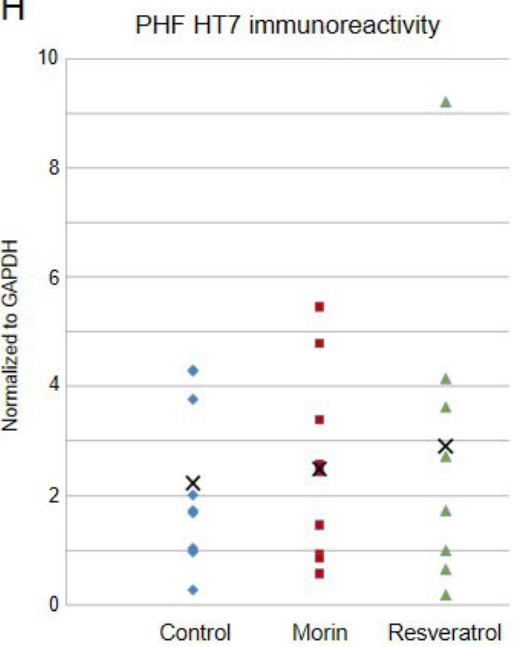

F

Homogenate AT8/HT7 immunoreactivity
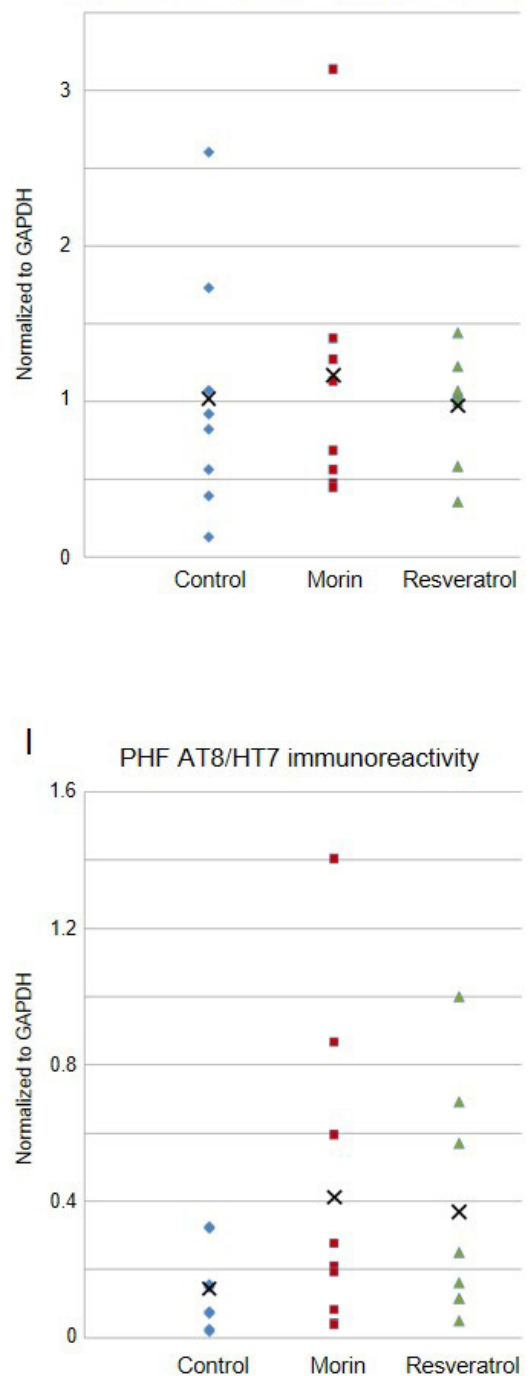

Figure 2. Tau in immunoblots. A) Representative AT8 antibody immunoblot of whole brain homogenate, showing hyperphosphorylated tau. B) Representative HT7 antibody immunoblot of whole brain homogenate, showing total tau. C) Representative GAPDH antibody immunoblot of whole brain homogenate, indicating total protein. D) AT8 antibody immunoreactivity in whole brain homogenate. Each point represents one mouse. Values for some mice were nearly the same, thus some points overlap. " $\mathrm{X}$ " represents the mean value for each drug. E) HT7 antibody immunoreactivity in whole brain homogenate. F) Ratio of AT8 to HT7 antibody immunoreactivity in whole brain homogenate. G) AT8 antibody immunoreactivity in PHF. H) HT7 antibody immunoreactivity in PHF. I) Ratio of AT8 to HT7 antibody immunoreactivity in PHF. 
A

AT8 immunoreactivity

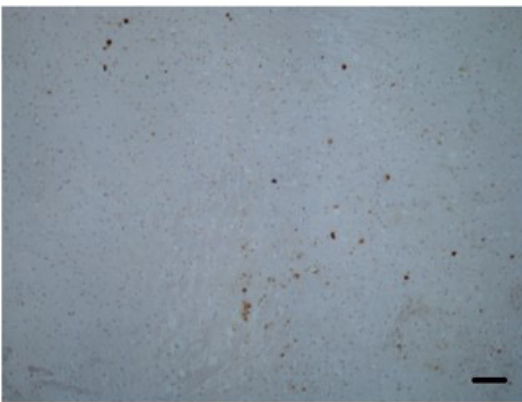

Control

B

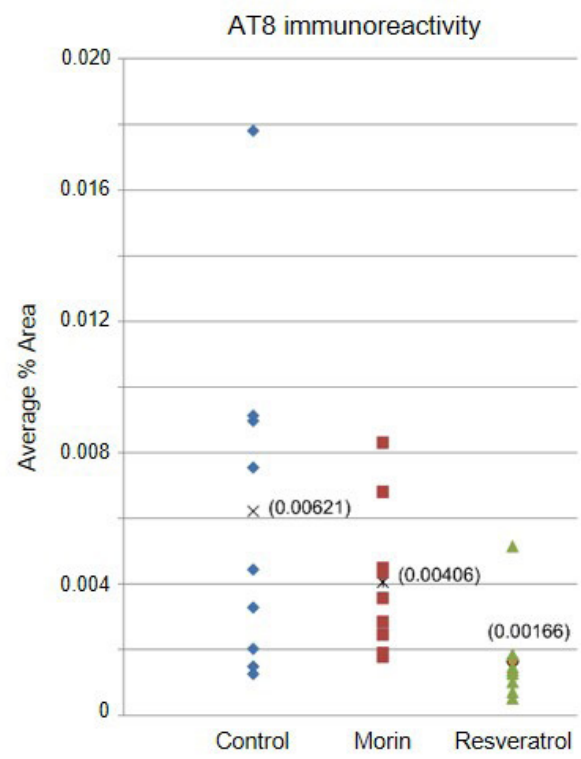

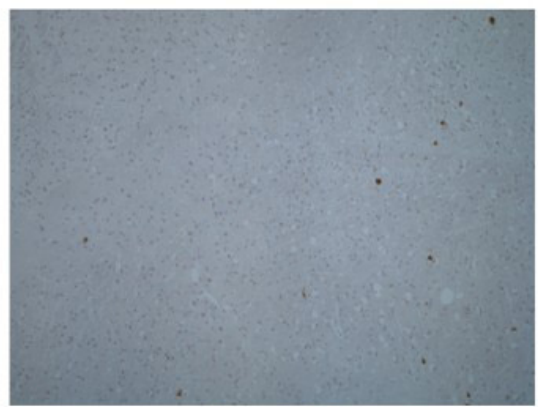

Resveratrol

C

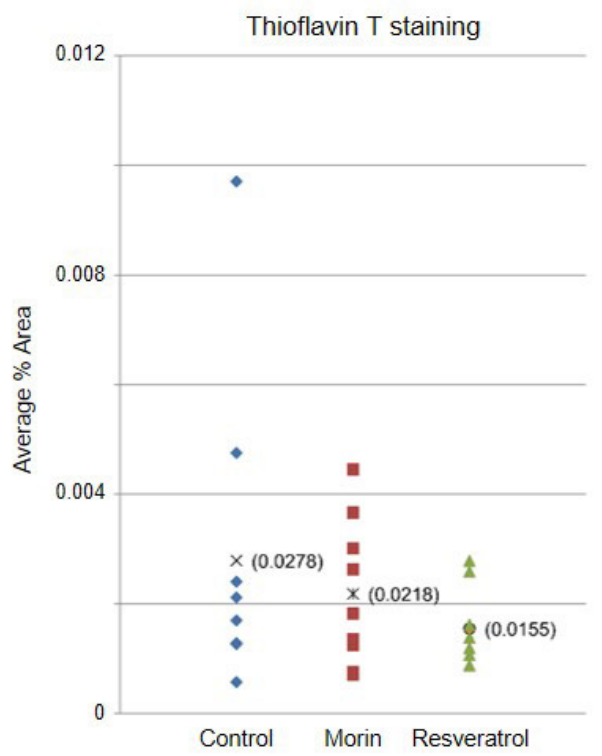

Figure 3. Hyperphosphorylated tau in brain sections. A) Representative photos of sections stained by AT8 antibody. Brown indicates phosphorylated tau stained by AT8. Blue indicates cell nuclei stained by hematoxylin. Scale bar: $50 \mu \mathrm{m}$. B) Effect of drug treatment on the area of sections stained by AT8 antibody. Each point represents one mouse. Values for some mice were the same, thus some points overlap. For each drug, " $\mathrm{X}$ " represents the mean value, shown in parentheses. $\mathrm{C}$ ) Effect of drug treatment on the area of sections stained by Thioflavin T.

decreasing soluble hyperphorylated tau. To understand these findings, these compounds can be incubated with aggregated recombinant tau or with brain homogenate from tanglecontaining transgenic mice or postmortem human brain tissue.

If these compounds or derivatives could be used to reduce tau hyperphosphorylation or aggregation, they may have potential to treat $A D$ and other tauopathies, such as Pick's disease, corticobasal degeneration, progressive supranuclear palsy, or frontotemporal dementia and parkinsonism linked to chromosome 17 [21]. Although tangles may not in and of themselves be neurotoxic [2] (in fact, suppression of mutant tau expression can restore brain function despite continued tangle deposition [22]), they may serve as indicators that neurotoxic forms of tau had been present. Thus, treatments that lead to reductions in tangle density might also affect tau neurotoxicity. Further studies of resveratrol, morin, and related compounds in animal models of tauopathy are warranted to explore doses of drugs and ages of mice to explore possible conditions for treatment.

Two previous studies have investigated the effect of resveratrol on tau in mice $[15,16]$. Porquet et al found a reduction in the ratio of ptau to tau, however Varamini et al did not 
A

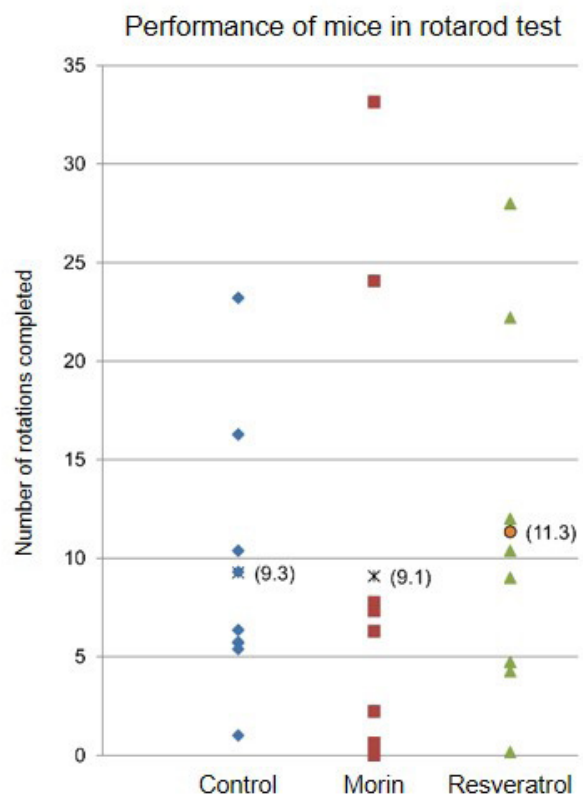

Figure 4. Rotarod test performance. Each point represents one mouse. Values for some mice were the same, thus some points overlap. Mean values are shown in parentheses.

$[15,16]$. Table 1 summarizes key features of these studies in an attempt to identify the cause of this discrepancy. Several factors were similar among studies and thus can likely be ruled out. The doses of resveratrol were comparable, as were the assay methods of Porquet et al and Varamini et al. Treatment began much later, and continued for a much shorter duration, in the study by Varamini et al than in the study by Porquet et al, which might have diminished any effect in the former study; however, our study encompassed an age range similar to that of Varamini et al yet detected an effect of resveratrol on ptau deposition. The number of mice tested by Varamini et al was smaller than in the other two studies if the wildtype and transgenic mice are counted separately. However, Figure 6 of Varamini et al displays very similar levels of ptau/tau for wildtype and transgenic mice, thus it may be reasonable to consider combining wildtype and transgenic mice for analysis, which would bring the number of mice up to the levels of the other studies. One factor differing among the studies was the animal model. Ours used human tau, Porquet et al used a strain of mice exhibiting premature aging, and Varamini et al used wildtype mice and mice overexpressing $A \beta$. It is not clear why resveratrol would affect tau differently in any of these strains, but perhaps this is part of the reason for the different effects among the three studies.

Our study showed that resveratrol and morin affected ptau accumulation yet did not affect motor function, as measured by our rotarod test. Perhaps treatment started after degeneration had already occurred, and functional loss was irreversible. Alternatively, the reduction of tangles and increase of soluble ptau by the compounds may not have fully suppressed disruption of normal tau function and resulting neurotoxicity.

\section{Conclusions}

In conclusion, in an animal study, resveratrol or morin tended to increase hyperphosphorylated tau on immunoblots but to decrease tangles in brain sections. Neither drug affected motor function.

\section{Acknowledgments}

Funding: This work was supported by Chinese University of Hong Kong Direct Grant for Research 2005.2.014. There are no potential financial, personal or professional conflicts of interest.

Table 1: Comparison of studies of the effect of resveratrol on tau in mice

\begin{tabular}{|c|c|c|c|c|}
\hline Factor & Porquet [15] & Varamini [16] & \multicolumn{2}{|c|}{ Yu (this paper) } \\
\hline Model & SAMP8 & wildtype; APP/PS & \multicolumn{2}{|c|}{ JNPL3 } \\
\hline \# of mice & $10-12$ v. $10-12$ & 3 v. $6 ; 6$ v. 3 & \multicolumn{2}{|c|}{9 v. 8} \\
\hline Age & 2 months & 12 months & \multicolumn{2}{|c|}{11 months } \\
\hline Duration & 7 months & 4 months & \multicolumn{2}{|c|}{3 months } \\
\hline Dose & $\sim 0.09 \mathrm{mg} / \mathrm{g}$, daily & $\sim 0.17 \mathrm{mg} / \mathrm{g}$, daily & \multicolumn{2}{|c|}{$0.10 \mathrm{mg} / \mathrm{g}, 5$ times $/ \mathrm{wk}$} \\
\hline Assay & ptau v. tau blot & ptau v. tau blot & ptau sections & ptau v. tau blot \\
\hline Fraction & $10 \mathrm{~kg}$ supernatant & $13 \mathrm{~kg}$ supernatant & all & PHF \\
\hline Effect & Decrease & no change & decrease & no change \\
\hline
\end{tabular}


[1] Iqbal K, Liu F, Gong CX, Alonso AC, Grundke-lqbal I. Mechanisms of tau-induced neurodegeneration. Acta Neuropathol 2009;118:53-69.

[2] Spires-Jones TL, Kopeikina KJ, Koffie RM, de Calignon A, Hyman BT. Are tangles as toxic as they look? J Mol Neurosci 2011;45:438-44.

[3] Rang HP, Dale MM. Rang and Dale's Pharmacology. Churchill Livingstone; 2007.

[4] Pillai JA, Cummings JL. Clinical trials in predementia stages of Alzheimer disease. Med Clin North Am 2013;97:439-57.

[5] Liu L, Drouet V, Wu JW, Witter MP, Small SA, Clelland C, et al. TransSynaptic Spread of Tau Pathology In Vivo. PLoS One 2012;7:e31302.

[6] Lee VM, Brunden KR, Hutton M, Trojanowski JQ. Developing therapeutic approaches to tau, selected kinases, and related neuronal protein targets. Cold Spring Harb Perspect Med 2011;1:a006437.

[7] Brunden KR, Trojanowski JQ, Lee VM. Advances in tau-focused drug discovery for Alzheimer's disease and related tauopathies. Nat Rev Drug Discov 2009;8:783-93.

[8] Gong EJ, Park HR, Kim ME, Piao S, Lee E, Jo DG, et al. Morin attenuates tau hyperphosphorylation by inhibiting GSK3beta. Neurobiol Dis 2011;44:223-30

[9] Masuda M, Suzuki N, Taniguchi S, Oikawa T, Nonaka T, Iwatsubo T, et al. Small molecule inhibitors of alpha-synuclein filament assembly. Biochemistry (NY) 2006;45:6085-94.

[10] Taniguchi S, Suzuki N, Masuda M, Hisanaga S, Iwatsubo T, Goedert $M$, et al. Inhibition of heparin-induced tau filament formation by phenothiazines, polyphenols, and porphyrins. J Biol Chem 2005;280:7614-23.

[11] Marambaud P, Zhao H, Davies P. Resveratrol promotes clearance of Alzheimer's disease amyloid-beta peptides. J Biol Chem 2005;280:37377-82.

[12] Anekonda TS. Resveratrol--a boon for treating Alzheimer's disease? Brain Res Rev 2006;52:316-26.

[13] Vingtdeux V, Dreses-Werringloer U, Zhao H, Davies P, Marambaud P. Therapeutic potential of resveratrol in Alzheimer's disease. BMC
Neurosci 2008;9 Suppl 2:S6.

[14] Karuppagounder SS, Pinto JT, Xu H, Chen HL, Beal MF, Gibson GE. Dietary supplementation with resveratrol reduces plaque pathology in a transgenic model of Alzheimer's disease. Neurochem Int 2009;54:111-8.

[15] Porquet D, Casadesus G, Bayod S, Vicente A, Canudas AM, Vilaplana J, et al. Dietary resveratrol prevents Alzheimer's markers and increases life span in SAMP8. Age (Dordr) 2013;35:1851-65.

[16] Varamini B, Sikalidis AK, Bradford KL. Resveratrol increases cerebral glycogen synthase kinase phosphorylation as well as protein levels of drebrin and transthyretin in mice: an exploratory study. Int J Food Sci Nutr 2014;65:89-96.

[17] Lewis J, McGowan E, Rockwood J, Melrose H, Nacharaju P, Van SM, et al. Neurofibrillary tangles, amyotrophy and progressive motor disturbance in mice expressing mutant (P301L) tau protein. Nat Genet 2000;25:402-5.

[18] Klunk WE, Wang Y, Huang GF, Debnath ML, Holt DP, Mathis CA. Uncharged thioflavin-T derivatives bind to amyloid-beta protein with high affinity and readily enter the brain. Life Sci 2001;69:1471-84.

[19] Biernat J, Mandelkow EM, Schroter C, Lichtenberg-Kraag B, Steiner $B$, Berling B, et al. The switch of tau protein to an Alzheimer-like state includes the phosphorylation of two serine-proline motifs upstream of the microtubule binding region. EMBO J 1992;11:1593-7.

[20] Chai X, Wu S, Murray TK, Kinley R, Cella CV, Sims H, et al. Passive immunization with anti-Tau antibodies in two transgenic models: reduction of Tau pathology and delay of disease progression. J Biol Chem 2011;286:34457-67.

[21] Spillantini MG, Goedert M. Tau pathology and neurodegeneration. Lancet Neurol 2013;12:609-22.

[22] Santacruz K, Lewis J, Spires T, Paulson J, Kotilinek L, Ingelsson M, et al. Tau suppression in a neurodegenerative mouse model improves memory function. Science 2005;309:476-81. 